\section{Reliability, Flexibility, And Environmental Impact Of Alternative Arterial Offset Optimization Objective Functions}

$\mathbf{J} \mathbf{R} \mathbf{P}$

Christopher M. Day ${ }^{1}$, Thomas M. Brennan Jr.1, Alexander M. Hainen ${ }^{1}$ Stephen M. Remias'1, Hiromal Premachandra ${ }^{1}$, James R. Sturdevant ${ }^{2}$

Greg Richards², Jason S. Wasson², and Darcy M. Bullock ${ }^{1}$
PuRdue

\begin{tabular}{lllllllllllllllll}
\hline$U$ & $N$ & $I$ & $V$ & $E$ & $R$ & $S$ & $I$ & $T$ & $Y$
\end{tabular}

Paper No. 11-0036

\section{Motivation}

There are a number of different opinions on which optimization objective function is best. It is desirable to use high resolution controller data and probe vehicle travel times to compare multiple
objective functions to determine which function performs best on a coordinated arterial?

\section{Approach}

Establish a baseline measurement of travel time along an arterial.

Optimize offset times using four alternative objective functions:

Minimize delay and stops

Maximize vehicle arrivals on green

Maximize vehicle arrivals on green without startup time

Implement four optimized offsets at eight intersections

Measure travel times of probe vehicles to assess travel time associaled with each objeclive.

Calculate the potential driver benefits for changing offset times to

accommodate optimal travel time and platoon dispersion.
Effect Of Adjusting Local Offset To Shift Vehicle Arrivals With Respect To Green Time

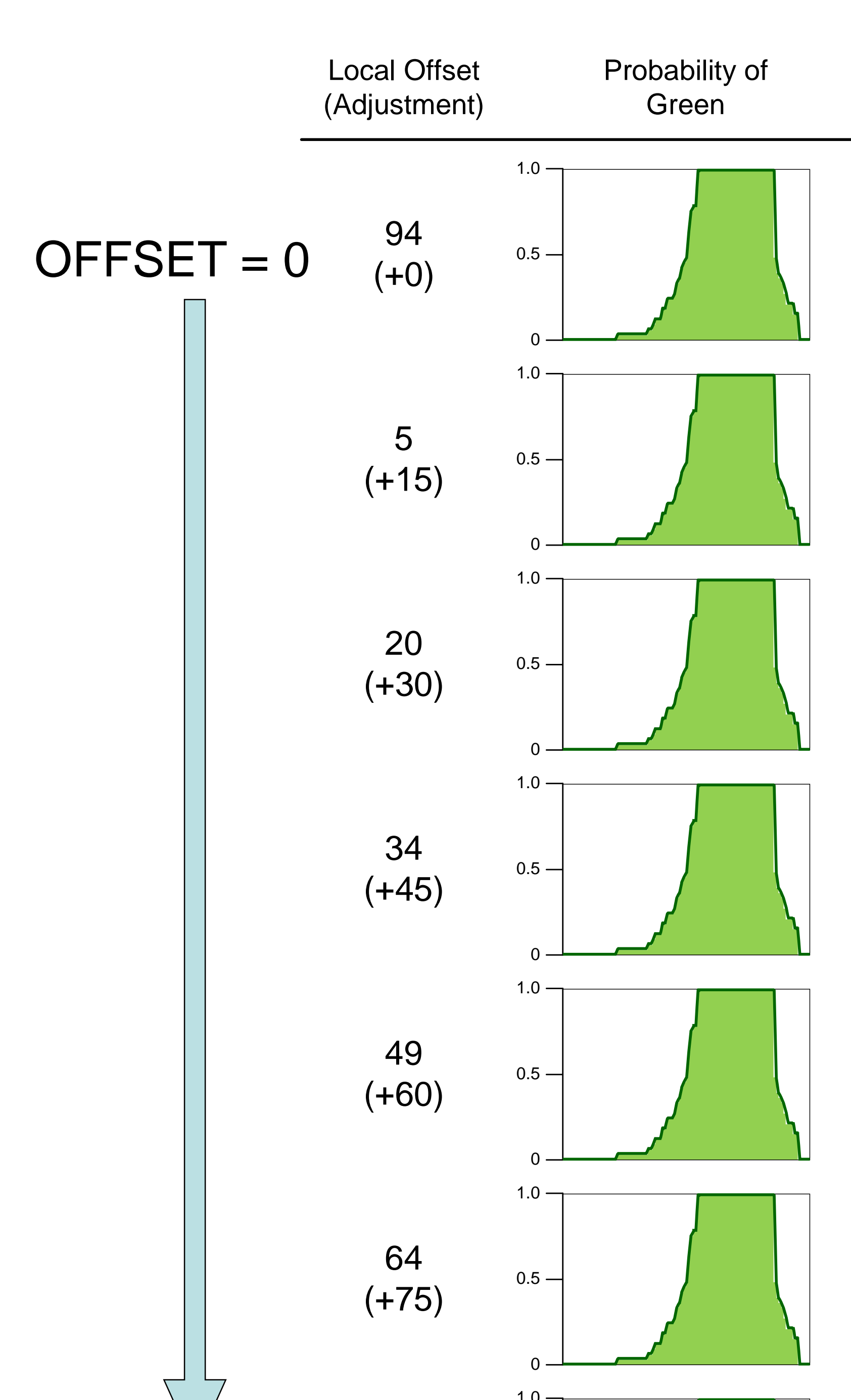

OFFSET $=90 \begin{gathered}79 \\ (+90)\end{gathered}$

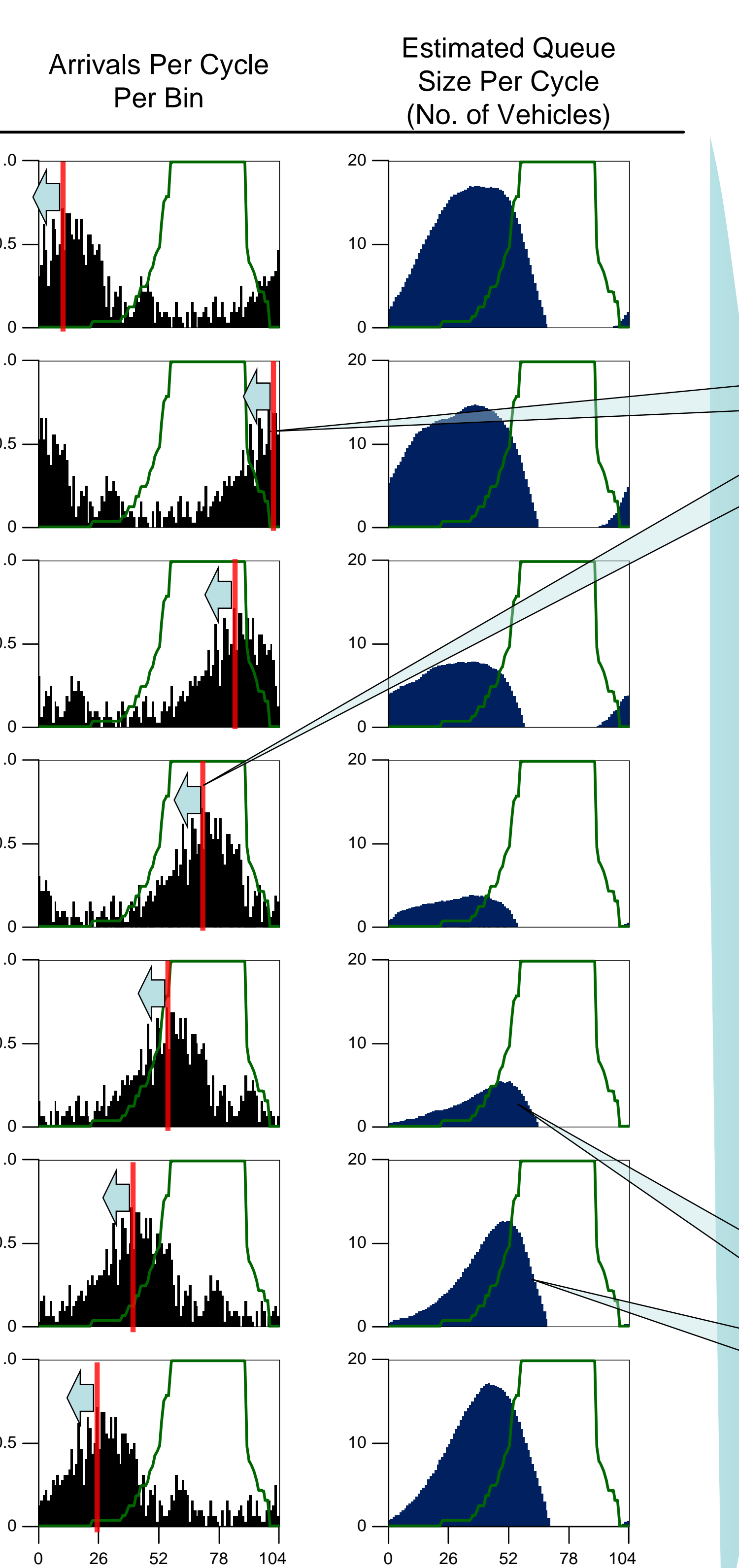

\section{Second shitsts
in venicic Arrivals}

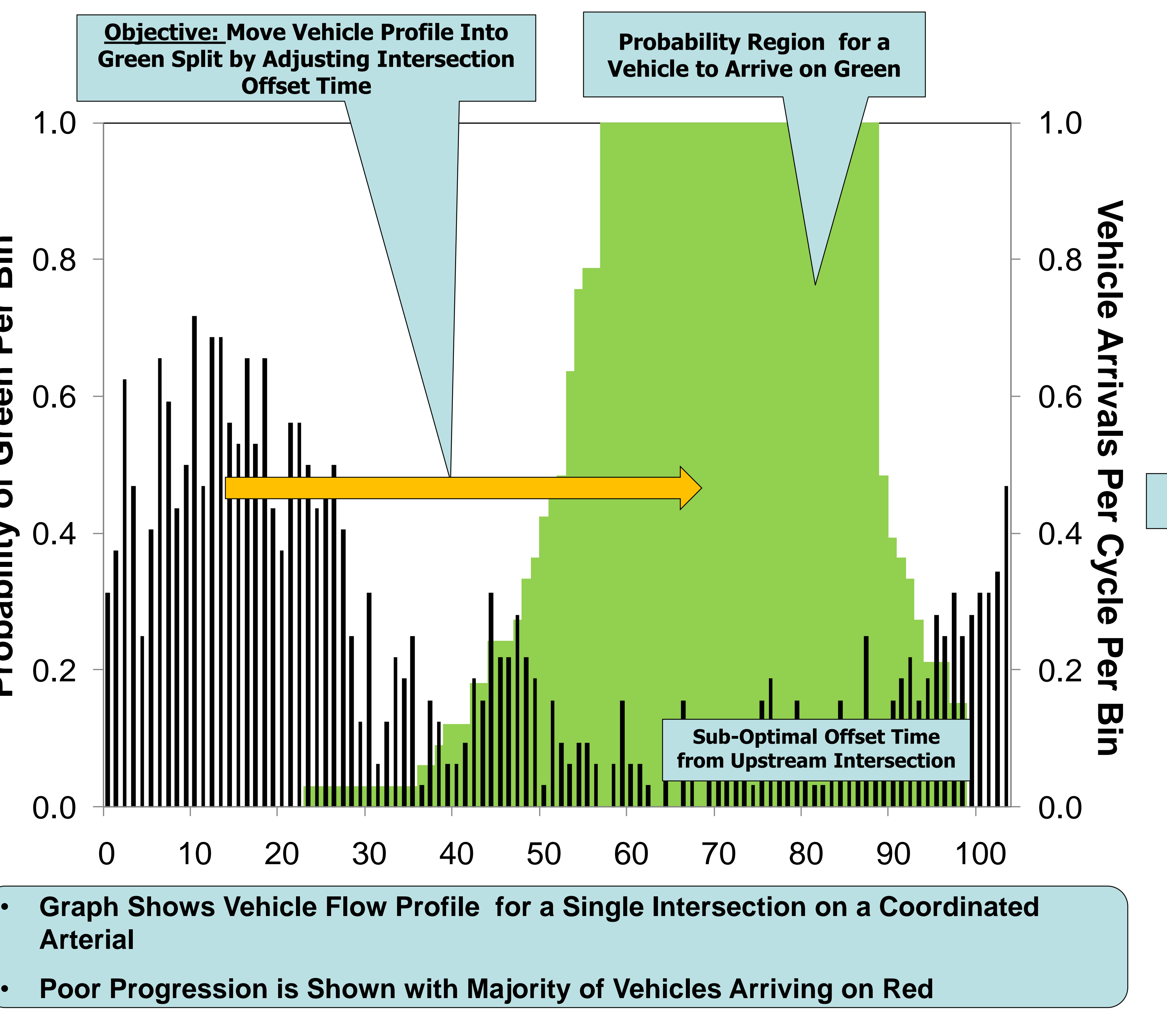

In General, Optimal Offsets Of Each Objective Functions Are Close
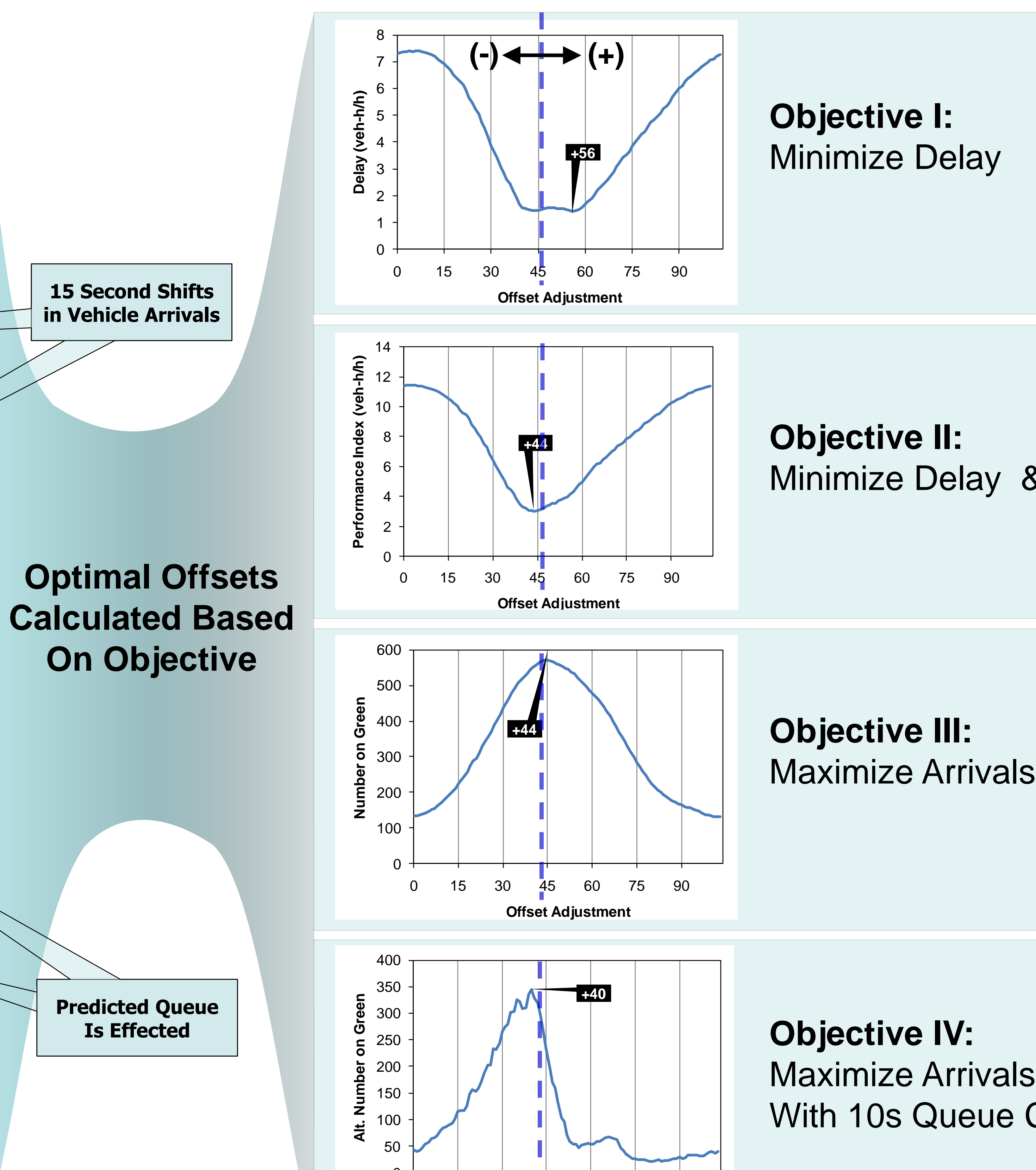

Offset Objective Function Concept

$$
\begin{array}{|l|}
\hline \text { - Objective I: Minimize delay (d) } \\
\qquad d=w \sum_{i} q_{i} \\
\qquad \begin{array}{l}
\text { - Objective II: Minimize delay and stops in } \\
\text { a performance index (PI). }
\end{array} \\
\qquad N_{g}=d+k \sum_{i} S_{i} \\
\hline \begin{array}{l}
\text { Objective III: Maximize arrivals on green } \\
\left(\mathrm{N}_{\mathrm{g}}\right) \text { [From Pro-Tracts \& ACS/lite] }
\end{array} \\
\qquad \begin{array}{l}
\text { - Objective IV: Maximize arrivals on green } \\
\text { with queue clearance time set at } 10 \\
\text { seconds }
\end{array} \\
\hline
\end{array}
$$

Calculated Optimal Offsets Tested On Weekend Plans

Along Study Corridor
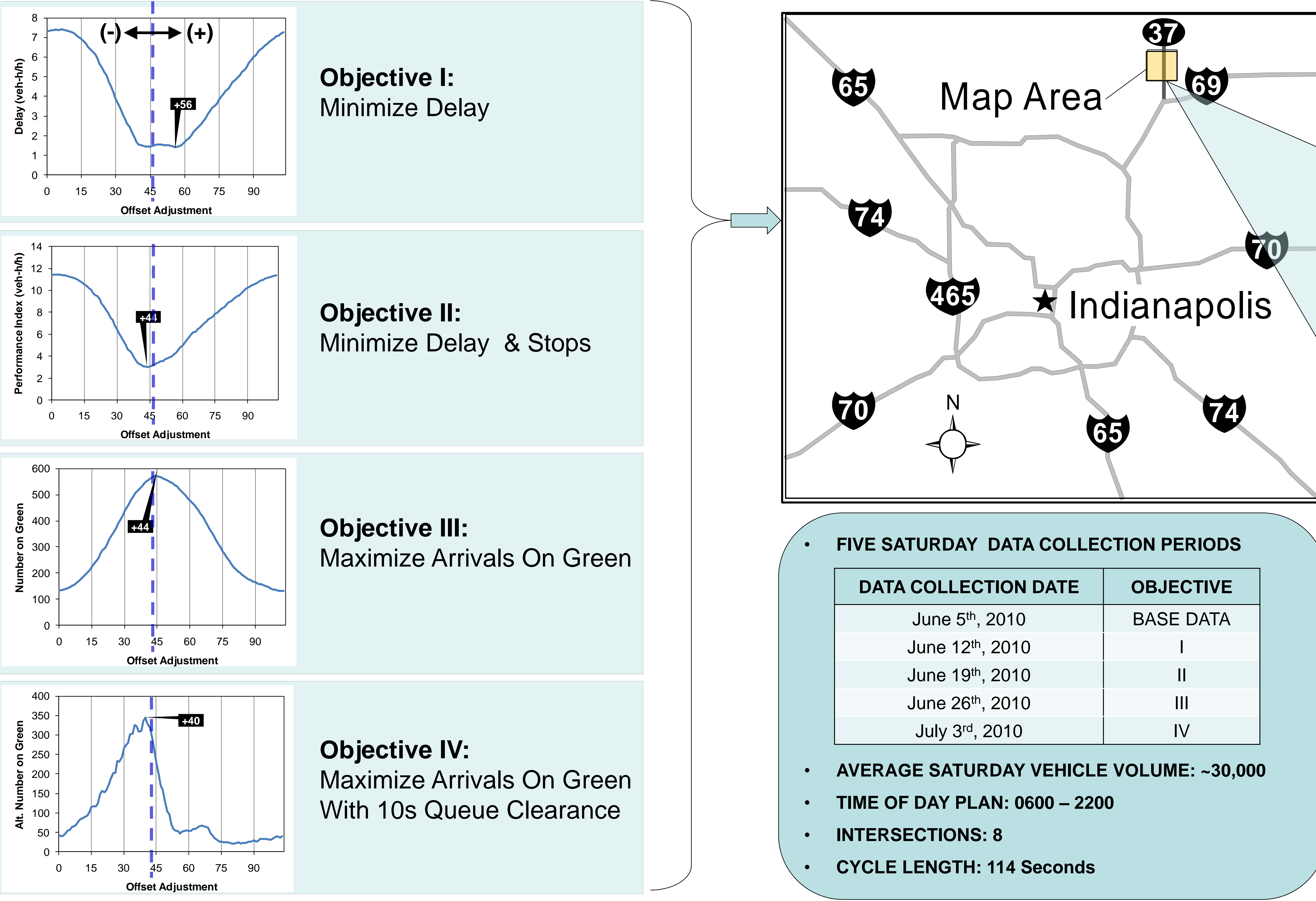

\section{Objective III:}

Maximize Arrivals On Green

Objective II: Minimize Delay \& Stops

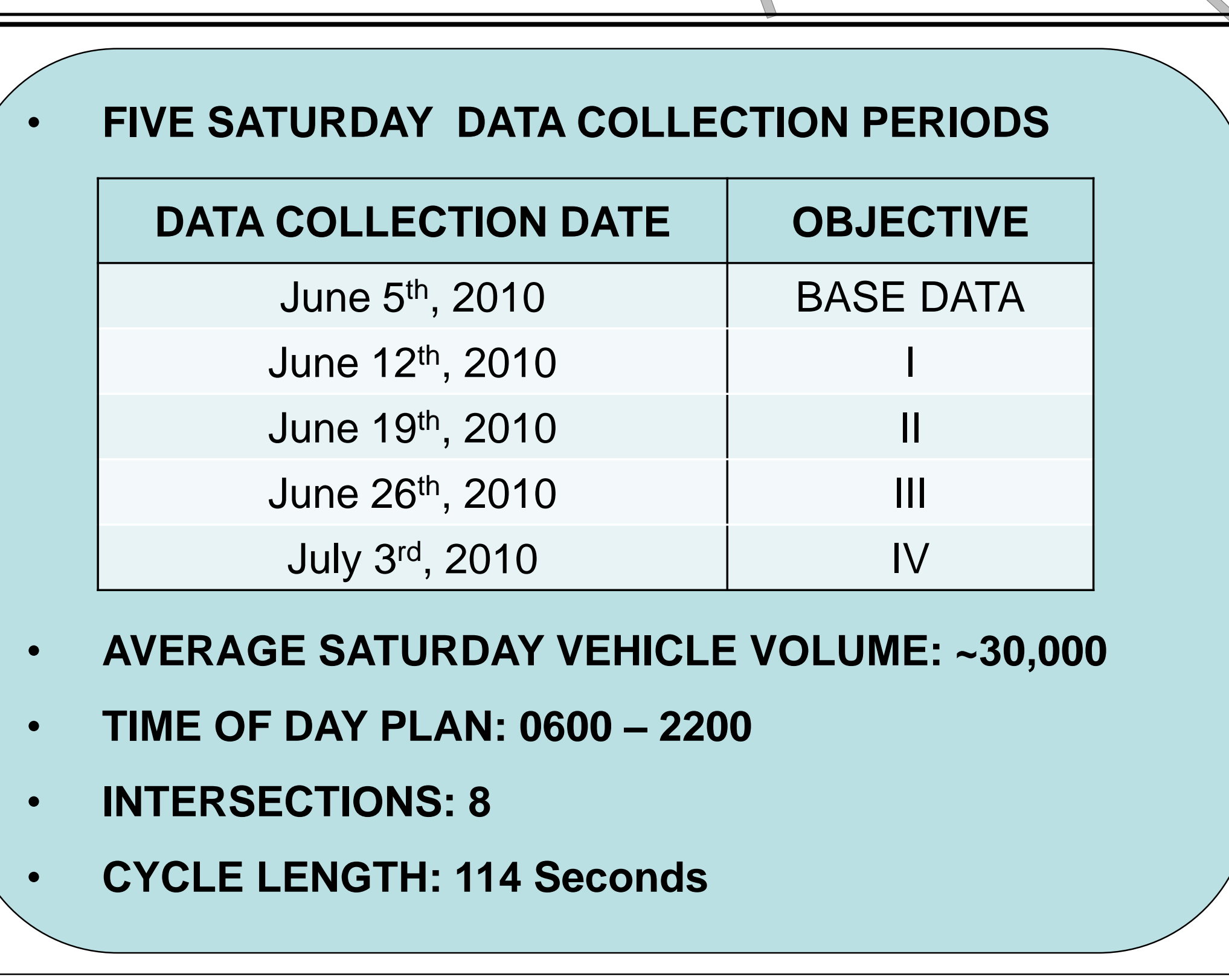

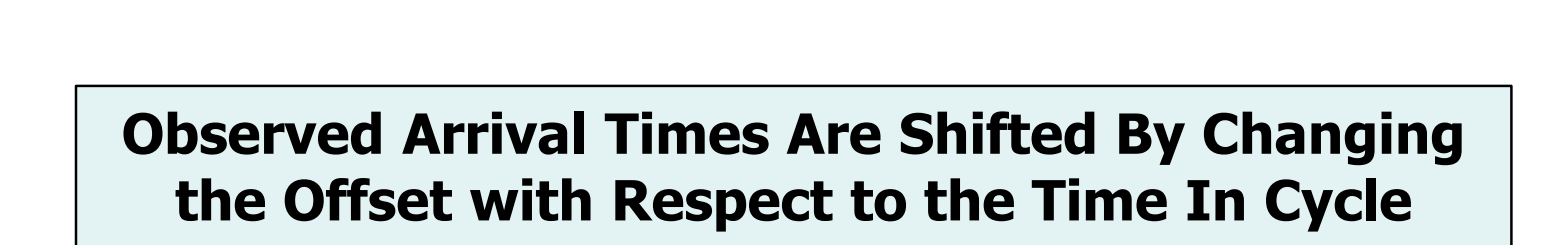
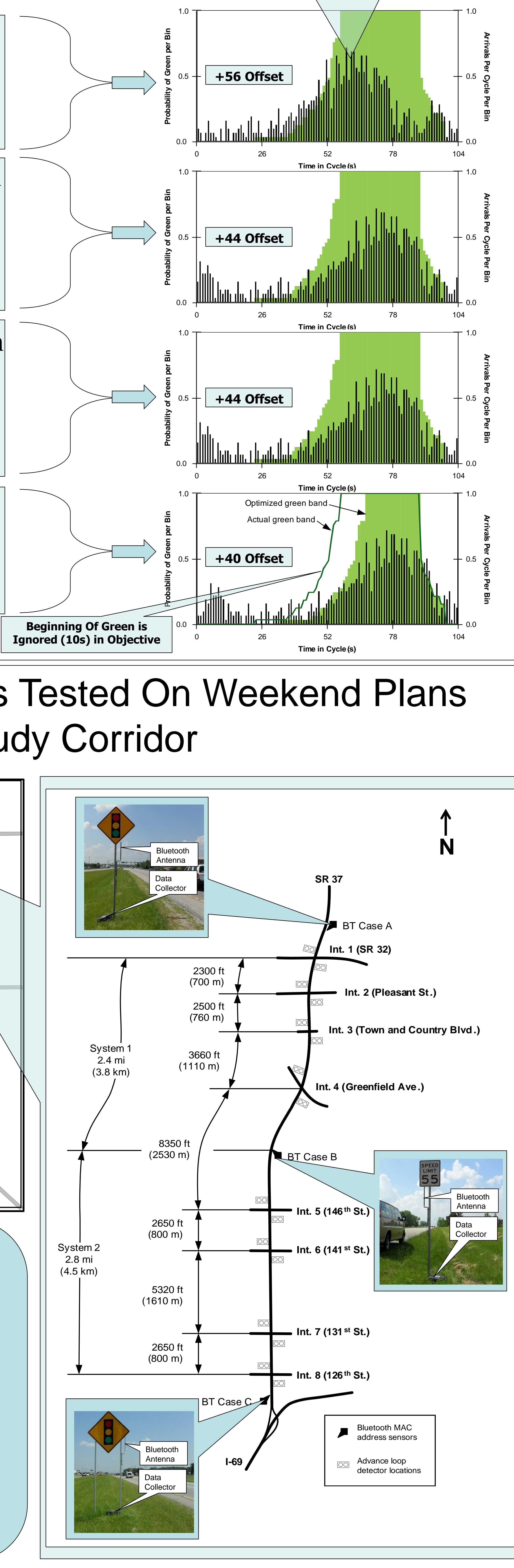


\section{Reliability, Flexibility, And Environmental Impact Of Alternative Arterial Offset Optimization Objective Functions}

Christopher M. Day ${ }^{1}$, Thomas M. Brennan Jr. ${ }^{1}$, Alexander M. Hainen ${ }^{1}$, Stephen M. Remias ${ }^{1}$, Hiromal Premachandra ${ }^{1}$, James R. Sturdevant ${ }^{2}$ Greg Richards ${ }^{2}$, Jason S. Wasson ${ }^{2}$, and Darcy M. Bullock' ${ }^{1}$

\section{PURDUE}

\begin{tabular}{llllllllll}
\hline$U$ & $N$ & $I$ & $V$ & $E$ & $R$ & $S$ & $I$ & $T$ & $Y$
\end{tabular}

Paper No. 11-0036
Flow Profiles For Baseline And Optimized Offsets For The Saturday (0600 - 1800) TOD Plan

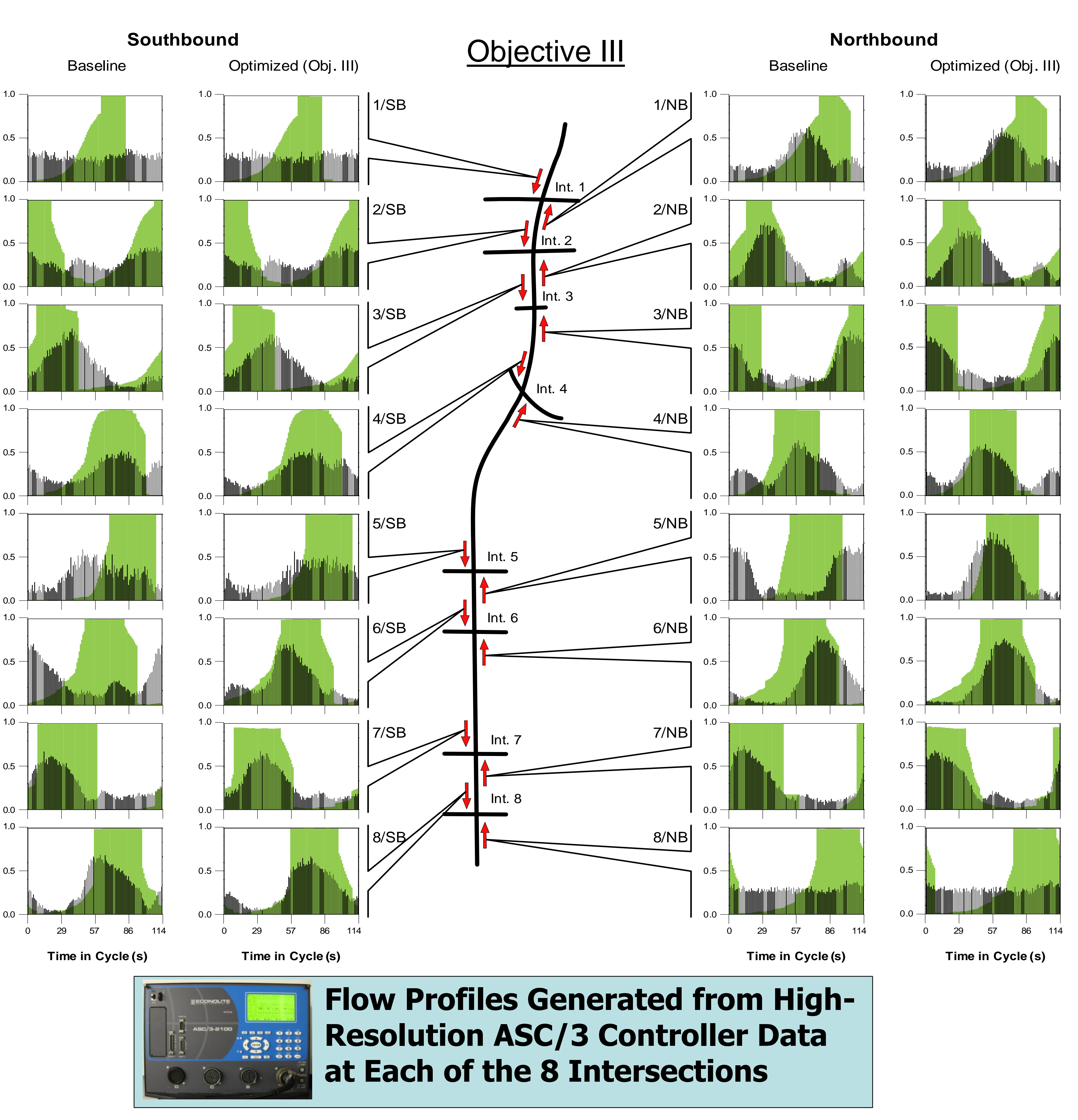

Measured Travel Times Along Corridor To Assess The Effect Of Offset Change
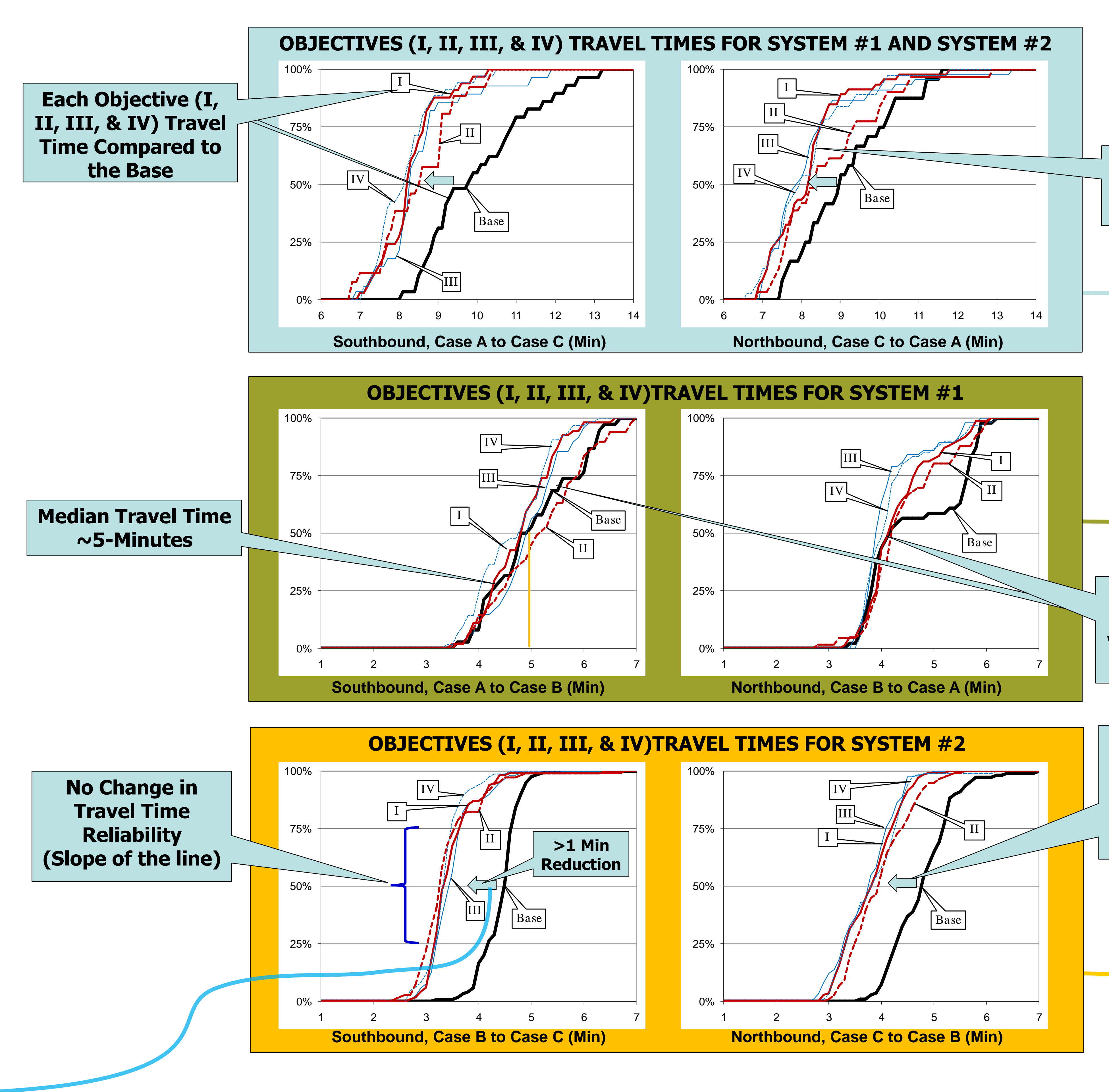
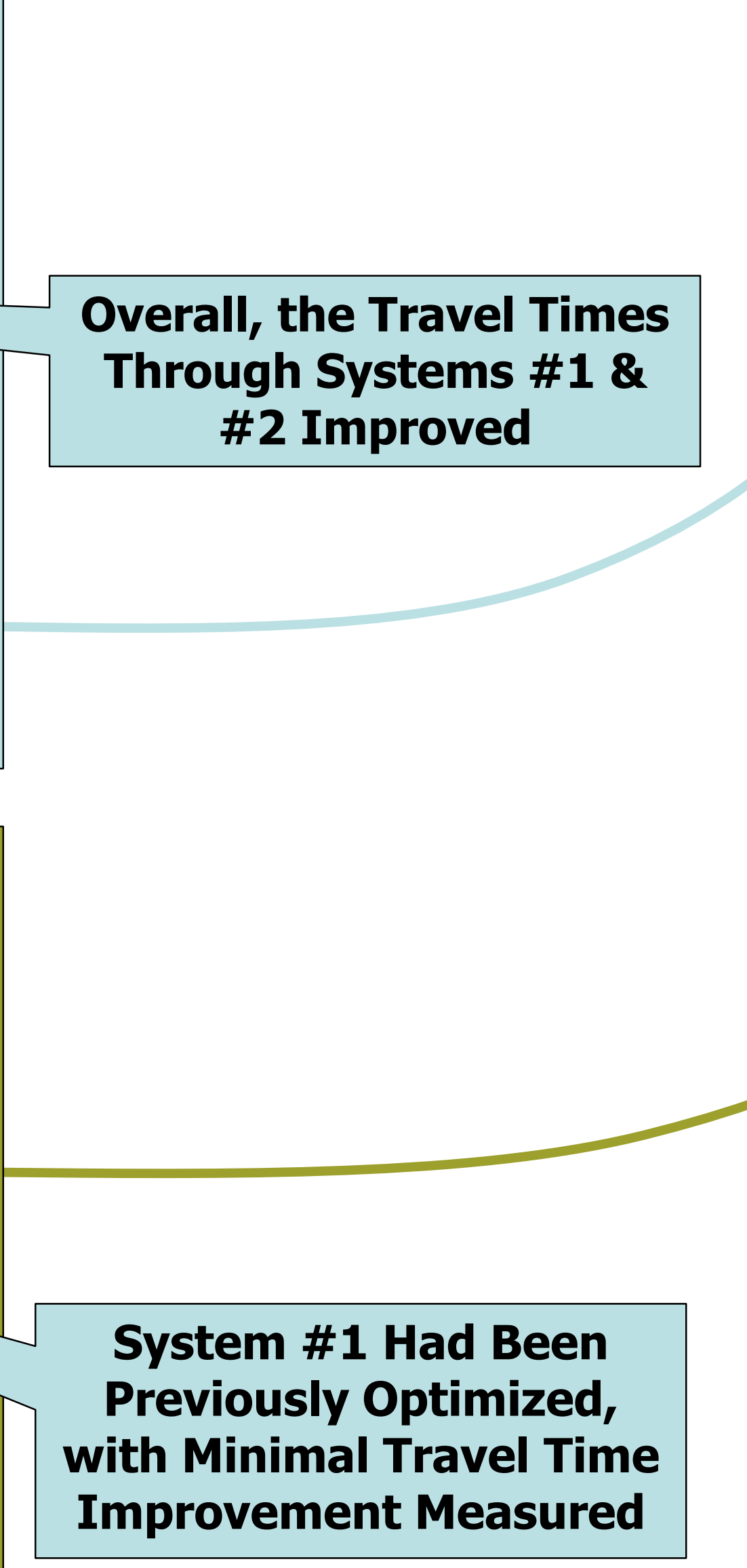

Improvement Measured

System \#2 had Not Been
Previously Optimized and Previously Optimized and
it Showed the Most Improvement to the
Travel Time

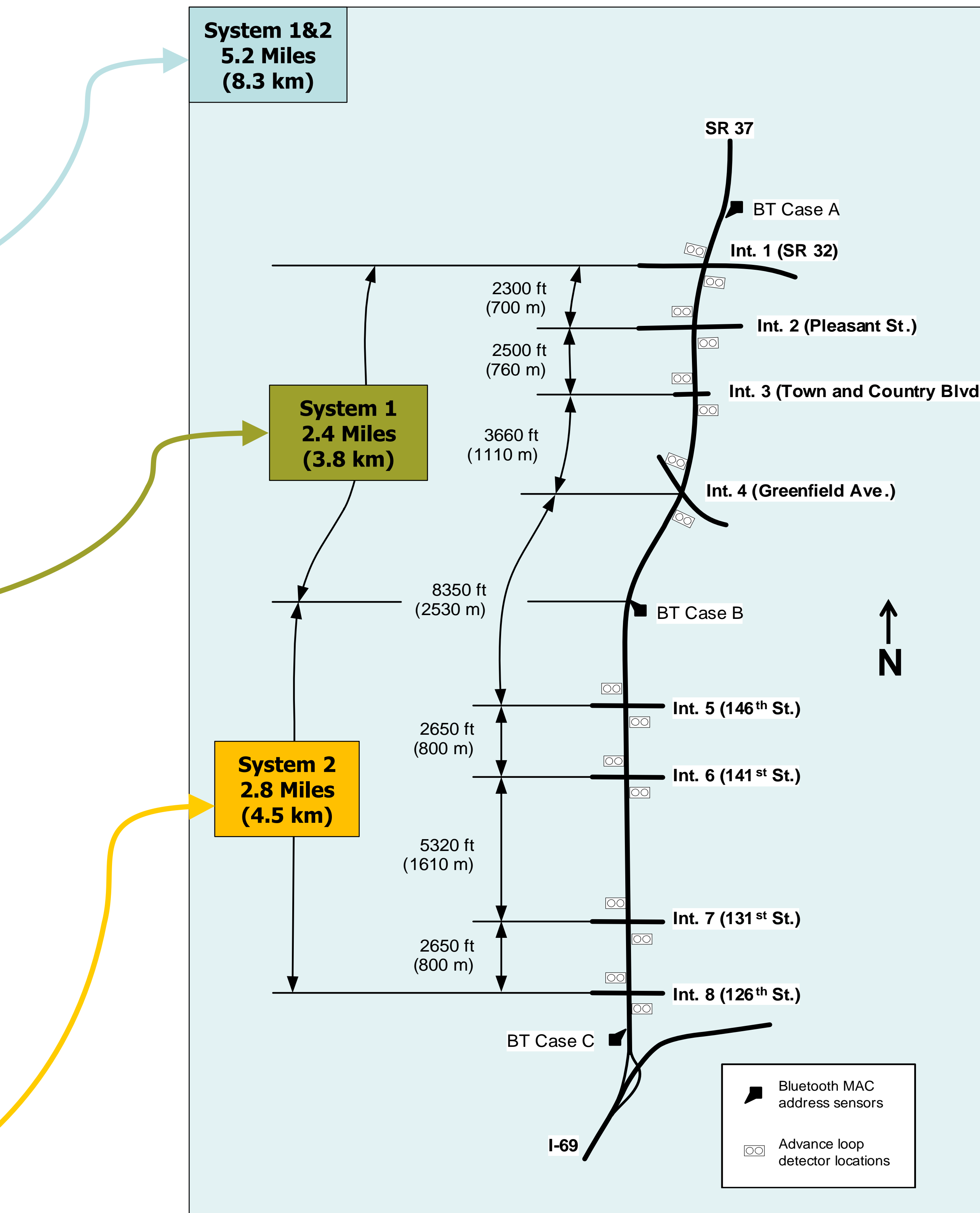

\section{OBJECTIVES (I, II, III, \& IV)TRAVEL TIMES FOR SYSTEM \#2}

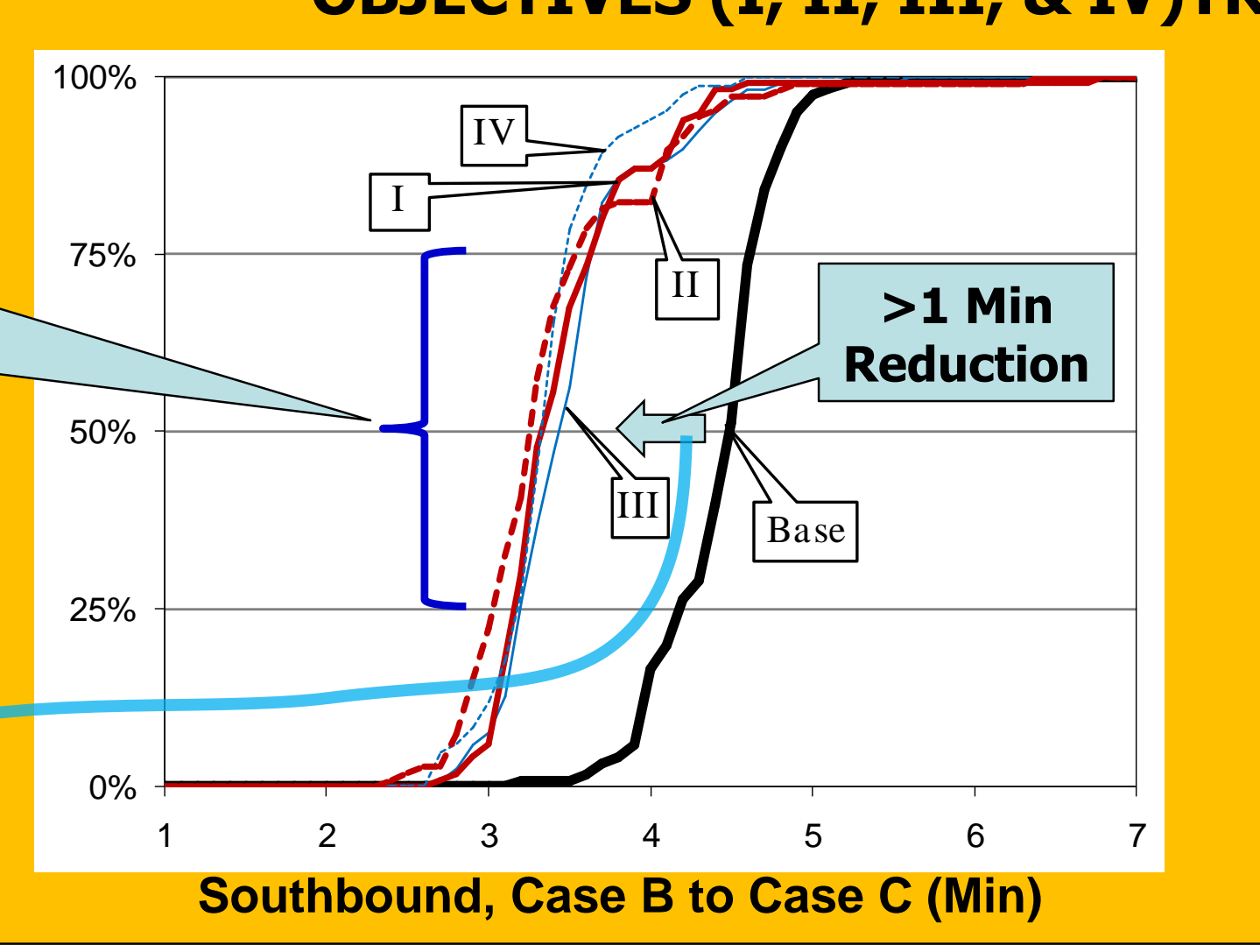

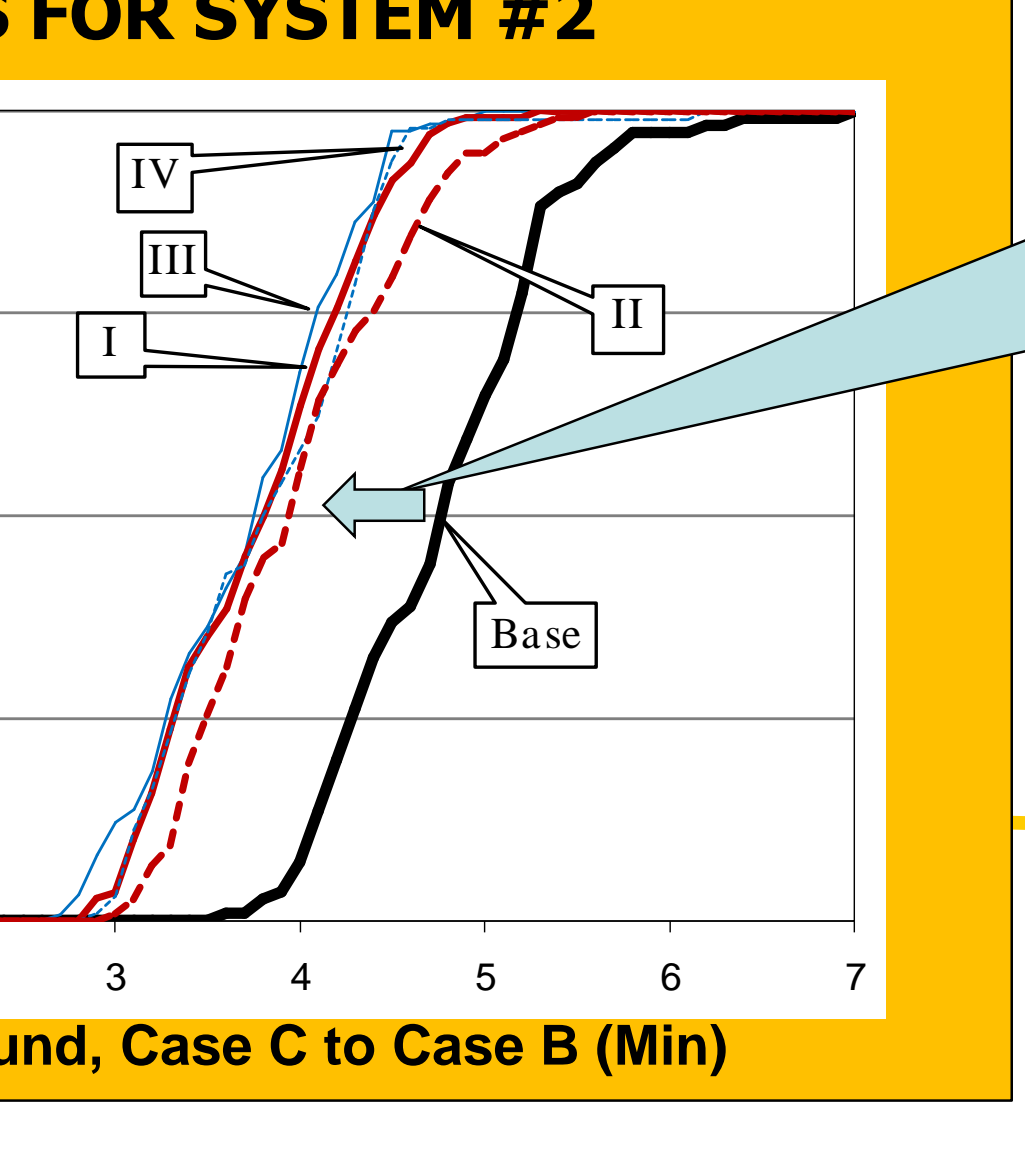
Travel Time

Northbound, Case C to Case B (Min)
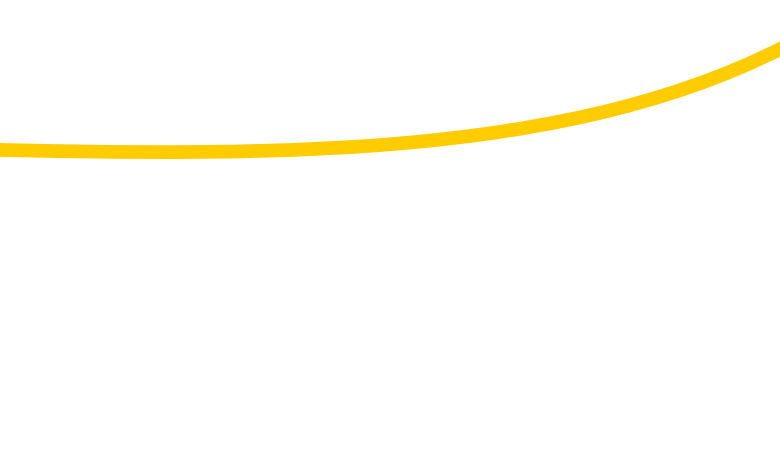

Travel Times Based For Each System and Each Objective
Syach

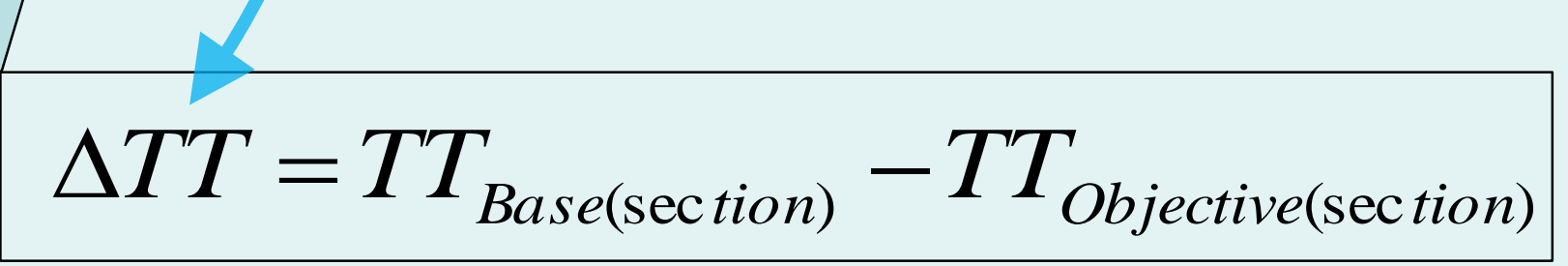

2\% Trucks: $U_{S E R}=\Delta T T * V o l * \% T * P P V_{t} * \frac{\$ 102.12}{\mathrm{hr}} * \frac{1 \mathrm{hr}}{60 \mathrm{~min}}$ 98\% Cars: $U S E R_{c}=\Delta T T * V o l * \% C * P P V_{c} * \frac{\$ 15.47}{\mathrm{hr}} * \frac{1 \mathrm{hr}}{60 \mathrm{~min}}$ $F U E L=\Delta T T * V o l * \frac{0.87 \mathrm{gal}}{\mathrm{hr}} * \frac{1 \mathrm{hr}}{60 \mathrm{~min}}$ $C O_{2}=F U E L * \frac{19.4 \mathrm{lbs}}{\text { gal }} * \frac{1 \mathrm{ton}}{2000 \mathrm{lbs}}$ $\mathrm{CC}=\mathrm{CO}_{2} * \frac{\$ 22}{\text { ton }}$

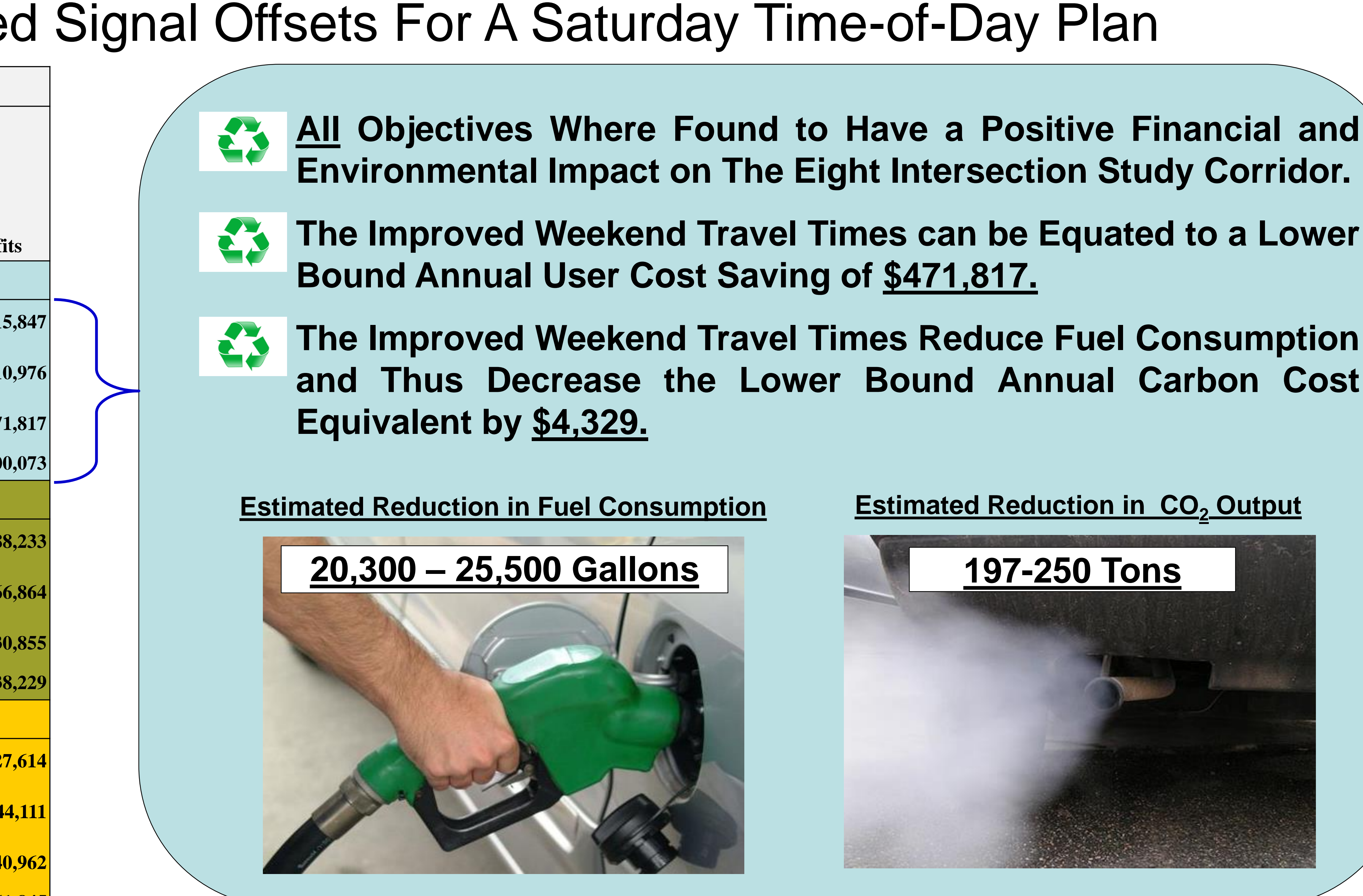

All Objectives Where Found to Have a Positive Financial and

$\stackrel{\text { Daily }}{\mathrm{CO}_{2}} \quad \frac{\mathrm{Anmual}}{\mathrm{CO}_{2}}$

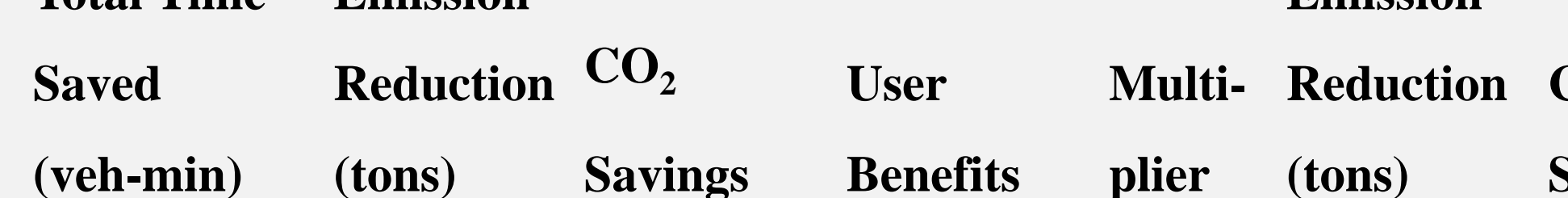

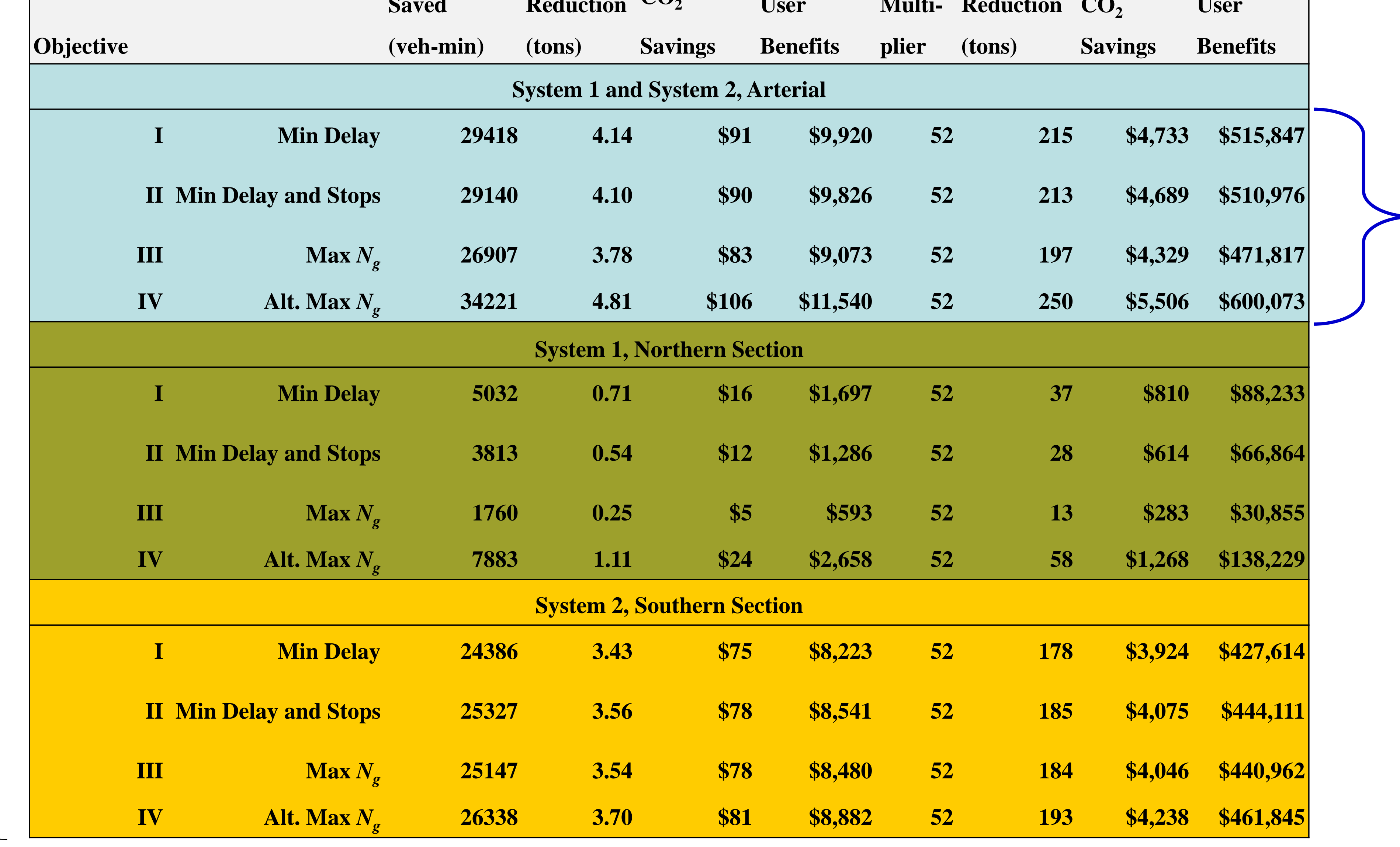

